\title{
$\mathrm{gl}(\lambda)$ and Differential Operators Preserving Polynomials
}

\section{GERHARD POST}

Department of Applied Mathematics, University of Twente, PO Box 217, 7500 AE Enschede,

The Netherlands

\section{NICO VAN DEN HIJLIGENBERG}

Center for Mathematics and Computer Science, PO Box 94079, 1090 GB Amsterdam, The Netherlands

(Received: 20 November 1995)

\begin{abstract}
We discuss a certain generalization of $\mathrm{gl}_{n}(\mathbb{C})$, and show how it is connected to polynomial differential operators that leave the polynomial space $\mathbb{P}_{n}$ invariant.
\end{abstract}

Mathematics Subject Classifications (1991). 17B66, 17B56, 17B35.

Key words: Lie algebras, differential operators, deformations, universal enveloping algebra, $\operatorname{sl}_{2}(\mathbb{C})$, $\mathrm{gl}(\lambda)$.

\section{Introduction}

Our aim is to study a certain Lie algebra, named $\operatorname{gl}(\lambda)$, and to show its relation to linear differential operators that leave the space $\mathbb{P}_{n}$ invariant. Here, $\mathbb{P}_{n}$ denotes the space of polynomials up to degree $n$.

The name $\operatorname{gl}(\lambda)$ is given to this Lie algebra by Feigin [4]. The reason for this is the following: if $\lambda \in\{2,3,4,5, \ldots\}$, then $\operatorname{gl}(\lambda)$ contains an ideal, which we denote by $I_{\lambda}$. The quotient $\mathrm{gl}(\lambda) / I_{\lambda}$ is isomorphic to $\mathrm{gl}_{\lambda}(\mathbb{C})$.

It will turn out that $\mathrm{gl}(\lambda)$ and $\mathrm{gl}(-\lambda)$ are isomorphic. If one takes $\lambda \in \mathbb{C}$, $\lambda \notin\{ \pm 2, \pm 3, \pm 4, \ldots\}, \operatorname{gl}(\lambda)$ has a one-dimensional center, denoted by $\langle 1\rangle$, and $\operatorname{sl}(\lambda):=\operatorname{gl}(\lambda) /\langle 1\rangle$ is simple. In this sense, $\operatorname{gl}(\lambda)$ and $\operatorname{sl}(\lambda)$ are infinite-dimensional generalizations of $\mathrm{gl}_{n}(\mathbb{C})$ and $\operatorname{sl}_{n}(\mathbb{C})$.

The Lie algebra $\mathrm{gl}(\lambda)$ can be defined in several ways. Here we will discuss three ways of doing it, but mostly we will work with the realization in terms of differential operators. The advantage of this approach is that it allows explicit calculations (notably the 2-cocycles $c_{2}^{\prime}$ and $c_{2}^{\prime \prime}$ defined below), whereas in the other two ways of defining $\operatorname{gl}(\lambda)$, this is (even) more intricate. Moreover, the realization in terms of differential operators, can be used to construct solutions of the Schrödinger equation $\Psi_{x x}+V(x) \Psi=E \Psi$, see, for example, [11].

The algebra $\operatorname{gl}(\lambda)$ appeared in the literature before. Dixmier [2] considered the structure of $\operatorname{gl}(\lambda)$ as an associative algebra, and proved that $\operatorname{gl}(\lambda)$ and $\operatorname{gl}\left(\lambda^{\prime}\right)$ 
are isomorphic if and only if $\lambda^{\prime}= \pm \lambda$. In [1], the same (using Dixmier's work) is proved for $\mathrm{gl}(\lambda)$ considered as Lie algebra.

The organization of this paper is as follows. Section 2 gives the definition of $\operatorname{gl}(\lambda)$ and some basic observations. Section 3 gives an equivalent definition (which is used in [2] and [1]). In Section 4, we take a closer look at $\mathrm{gl}(\lambda)$; in particular we prove that $\operatorname{sl}(\lambda)$ is simple for almost all $\lambda$. In Section 5 , we discuss the third way of defining $\operatorname{gl}(\lambda)$, namely as a deformation of the Poisson algebra of even polynomials on the plane. Finally, in Section 6, we discuss the relation between $\operatorname{gl}(\lambda)$ and the algebra of differential operators that preserve all polynomials up to a fixed degree.

We include proofs as far as space permits. For more details, we refer the reader to [9].

\section{Definition of $g 1(\lambda)$}

2.1.

Let $\mathfrak{D}$ denote the associative algebra of differential operators of the form

$$
T=\sum_{i=0}^{n} P_{i}(x) \frac{\mathrm{d}^{i}}{\mathrm{~d} x^{i}}, \quad P_{i} \in \mathbb{C}[x] .
$$

We will write $\partial^{i}$ instead of $\mathrm{d}^{i} / \mathrm{d} x^{i}$. The algebra $\mathfrak{D}$ has 2 structures which are very important for our considerations. The first structure is an increasing filtering, the order, which we denote by ord. We say that $T=\sum_{i=0}^{n} P_{i}(x) \partial^{i}$ has order $n$ (assuming that $P_{n} \neq 0$ ).

The second structure is a grading, the degree, denoted by deg. We say that $T \in \mathfrak{D}$ has degree $m$ if $T\left(x^{k}\right) \in\left\langle x^{k+m}\right\rangle$ for all $k \in \mathbb{N}$. Specifically, this means that $T$ has the form $T=\sum_{i=0}^{n} c_{i} x^{i+m} \partial^{i}$ for some constants $c_{i} \in \mathbb{C}$. The degree is an integer. If the degree is negative, say $\operatorname{deg}(T)=-m, m>0$, then the order of $T$ is at least $m$.

For two monomials $x^{k} \partial^{l}$ and $x^{i} \partial^{j}$ we say

$$
x^{k} \partial^{l} \geqslant x^{i} \partial^{j} \quad \text { if } l>j \text { or } l=j \text { and } k \geqslant i .
$$

If we write $x^{k} \partial^{l}+\cdots$ it will mean that the dots will contain only multiples of smaller monomials.

\section{2 .}

Now we turn to the definition of $\operatorname{gl}(\lambda)$. In $\mathfrak{D}$ we consider the (associative) subalgebra $\mathfrak{D}(\lambda)$, generated by 1 and

$$
e=x^{2} \partial-(\lambda-1) x, \quad h=2 x \partial-\lambda+1 \quad \text { and } f=-\partial .
$$


It would be more accurate to write $e_{\lambda}, h_{\lambda}$ and $f_{\lambda}$, but we will not do so. This algebra can be considered as a Lie algebra, by taking for the Lie product merely the commutator. The resulting Lie algebra is (by definition) $\operatorname{gl}(\lambda)$. We note that

$$
[e, f]=h, \quad[h, e]=2 e \quad \text { and } \quad[h, f]=-2 f
$$

for all $\lambda \in \mathbb{C}$, so $\langle e, f, h\rangle$ forms an $\operatorname{sl}_{2}(\mathbb{C})$-algebra. It means that polynomials in $e, h$ and $f$ can be expressed as a linear combination of $e^{i} h^{j} f^{k}$, for $i, j, k \in \mathbb{N}$ thanks to the Poincaré-Birkhoff-Witt theorem. Said differently, when looking for a linear basis of $\mathrm{gl}(\lambda)$, we can restrict to the terms $e^{i} h^{j} f^{k}$. Hence, the following lemma is easy to prove.

LEMMA 2.1. The Lie algebra $\operatorname{gl}(\lambda)$ has a basis of the form $x^{r} \partial^{s}+\cdots$ with $s=0,1,2, \ldots$ and $r \leqslant 2 s$.

Proof. Clearly these elements are linearly independent. Now

$$
e^{i} h^{j} f^{k}=(-1)^{k} 2^{j} x^{2 i+j} \partial^{i+j+k}+\cdots
$$

from which the lemma easily follows using the remark before.

This linear basis is independent of $\lambda$ only on top level (i.e. the term of highest order).

2.3 .

Let us introduce the following notation. The set of elements $T \in \mathfrak{D}$, such that $T\left(\mathbb{P}_{n}\right) \subset \mathbb{P}_{n}$, we denote by $\operatorname{Inv}\left(\mathbb{P}_{n}\right)$. It is obvious that if $\operatorname{deg}(T)=-m, m \geqslant 0$, then $T \in \operatorname{Inv}\left(\mathbb{P}_{n}\right)$, since $x^{k}$ is mapped to $x^{k-m}$. If $\mathfrak{D}_{-}$denotes the subalgebra of elements of degree 0 or less, then it is easy to see (using Lemma 2.1) that $\mathfrak{D}_{-}$is generated by $h$ and $f$ for all $\lambda \in \mathbb{C}$. Hence, $\mathfrak{D}_{-} \subset \operatorname{gl}(\lambda)$. How about $e$ ? It is clear that $\operatorname{deg}(e)=1$, so $e$ maps $x^{n}$ to a multiple of $x^{n+1}$. If this multiple is zero, e leaves $\mathbb{P}_{n}$ invariant. So let us examine it.

$$
e\left(x^{n}\right)=\left(x^{2} \partial-(\lambda-1) x\right)\left(x^{n}\right)=(n-(\lambda-1)) x^{n+1} .
$$

It follows that $e\left(x^{n}\right)=0$ if and only if $\lambda=n+1$. Clearly, in this case all elements of $\mathrm{gl}(\lambda)$ leave $\mathbb{P}_{n}$ invariant. So we have the following result.

PROPOSITION 2.2. For all $n \in \mathbb{N}$, there holds $\mathrm{gl}(n+1) \subset \operatorname{Inv}\left(\mathbb{P}_{n}\right)$.

Hence, for $n=0,1,2, \ldots$, we have a representation of $\mathrm{gl}(n+1)$ on $\mathbb{P}_{n}$. If $n=0$, this representation is trivial, as it means that $x^{2} \partial, x \partial$ and $\partial$ annihilate the space $\langle 1\rangle$, and only $1 \in \operatorname{gl}(\lambda)$ is represented as a nonzero element.

For $n=1,2, \ldots$ the situation is the following: $\mathbf{g l}(n+1)$ contains a nontrivial ideal being the kernel of this representation. Let us denote this ideal by $I_{n+1}$. All differential operators of order at most $n$ act nontrivial on $\mathbb{P}_{n}$. Again using Lemma 
2.1 we see that $\operatorname{dim}\left(\operatorname{gl}(n+1) / I_{n+1}\right)=1+3+5+\cdots+(2 n+1)=(n+1)^{2}$. So, comparing dimensions, we see that $\mathrm{gl}(n+1) /_{I_{n+1}} \simeq \mathrm{gl}_{n+1}(\mathbb{C})$ and therefore $\operatorname{sl}(n+1) / I_{n+1} \simeq \operatorname{si}_{n+1}(\mathbb{C})$.

\section{3. $\operatorname{gl}(\lambda)$ as Quotient Algebra of $\mathrm{U}\left(\mathrm{sl}_{2}(\mathbb{C})\right)$}

Now we describe $\mathrm{gl}(\lambda)$ as a quotient of $U=\mathrm{U}\left(\mathrm{sl}_{2}(\mathbb{C})\right)$, thereby providing a second description of $\operatorname{gl}(\lambda)$. Here $U$ is the universal enveloping algebra of $\operatorname{sl}_{2}(\mathbb{C})$. The algebra $\operatorname{gl}(\lambda)$ is generated by three elements, which span $\operatorname{sl}_{2}(\mathbb{C})$. Therefore, by a universal property of $U$, we know that $\operatorname{gl}(\lambda)$ is a quotient of $U$. We determine the ideal.

PROPOSITION 3.1. Let $J_{\lambda}$ denote the ideal in the associative algebra $U$ generated by

$$
\Delta-\left(\lambda^{2}-1\right), \quad \text { where } \Delta=2 e f+2 f e+h^{2} .
$$

Considered as Lie algebras, $U / J_{\lambda} \simeq \operatorname{gl}(\lambda)$.

Proof. By construction, $\operatorname{gl}(\lambda)$ is a quotient of $U$, say $\operatorname{gl}(\lambda)=U / Q_{\lambda}$. We need to prove that $Q_{\lambda}=J_{\lambda}$.

First, $Q_{\lambda} \supset J_{\lambda}$, since

$$
\begin{aligned}
2 e f+2 f e+h^{2}= & -2\left(x^{2} \partial+(\lambda+1) x\right) \partial-2 \partial\left(x^{2} \partial+\right. \\
& +(\lambda+1) x)+(2 x \partial+(\lambda+1))^{2}=\lambda^{2}-1 .
\end{aligned}
$$

To prove $Q_{\lambda} \subset J_{\lambda}$, one can compare dimensions using the natural filtering in $U$. Let $d_{n}$ denote the dimension of elements of filter $n$ (but not $n-1$ ). The Poincaré-Birkhoff-Witt theorem says that $d_{n}=\operatorname{dim}\left(S^{n}\right)$, where $S^{n}$ denotes the space of the $n$-fold symmetric tensors on $3=\operatorname{dim}\left(\mathrm{sl}_{2}(\mathbb{C})\right)$ elements. Hence, $d_{n}=\left(\begin{array}{c}n+2 \\ 2\end{array}\right)$. Again by the Poincaré-Birkhoff-Witt theorem it follows that the elements of the form

$$
e^{i} h^{j} f^{k}\left(\Delta-\left(\lambda^{2}-1\right)\right), \quad i, j, k \geqslant 0 .
$$

form a basis for $J_{\lambda}$. Hence $U / J_{\lambda}$ contains $\left(\begin{array}{c}n+2 \\ 2\end{array}\right)-\left(\begin{array}{c}n \\ 2\end{array}\right)=2 n+1$ linear independent elements of filter $n$, just like $\mathrm{gl}(\lambda)$. This completes the proof.

From this proposition, we derive a useful corollary.

COROLLARY 3.2. $\operatorname{gl}(\lambda) \simeq \operatorname{gl}(-\lambda)$

Proof.

$$
\Delta-\left(\lambda^{2}-1\right)=\Delta-\left((-\lambda)^{2}-1\right), \quad \text { so } \quad J_{\lambda}=J_{-\lambda} .
$$

In his article [4], Feigin takes the following definition for $\operatorname{gl}(\lambda)$ : he takes a Casimir $\tilde{\Delta}$ (which he does not specify) and defines $\operatorname{gl}(\lambda)$ to be the quotient of $U$ 
by $(\tilde{\Delta}-\lambda(\lambda-1) / 2)$. Casimirs are unique up to scaling and shift by a scalar, so $\tilde{\Delta}=\alpha \Delta+\beta$ for some $\alpha, \beta \in \mathbb{C}$. However, we were not able to match Feigin's definition with ours. In [2] also $U /(\Delta-\mu)$ is considered.

Let us make some remarks. Suppose that we have a nontrivial representation of $\operatorname{sl}_{2}(\mathbb{C})$ on a linear space $V$, so $\rho: \mathrm{sl}_{2}(\mathbb{C}) \rightarrow \operatorname{gl}(V)$, such that the Casimir $\Delta$ is represented by a scalar, say $\mu$. Such a representation can be considered as a representation for $\operatorname{gl}(\lambda)$ if $\lambda^{2}-1=\mu$. So let $\lambda$ satisfy this equation. Then one can ask for the kernel of $\rho$. In the next section, we will prove that $\operatorname{sl}(\lambda)$ is a simple Lie algebra if and only if $\lambda \notin\{ \pm 2, \pm 3, \pm 4, \ldots\}$. Therefore for $\lambda \notin\{ \pm 2, \pm 3, \pm 4, \ldots\}$ the kernel is $\{0\}$. In the other case, $\lambda \in\{ \pm 2, \pm 3, \pm 4, \ldots\}$, the ideal $I_{\lambda}$ as described above is the only ideal in $\operatorname{sl}(\lambda)$. Hence, the kernel of $\rho$ is either $\{0\}$ or $I_{\lambda}$. This gives a description of the primitive ideals [3] of $\mathrm{sl}_{2}(\mathbb{C})$, assuming that such an ideal contains $(\Delta-\mu)$ for some $\mu \in \mathbb{C}$. We remark that for infinite-dimensional representations $\rho(\Delta)$ is not always diagonalizable, not even for irreducible representations.

For finite-dimensional irreducible representations, $\Delta$ acts automatically as a scalar thanks to Schur's lemma. For $\mathrm{sl}_{2}(\mathbb{C})$ the situation is rather simple, namely $\operatorname{sl}_{2}(\mathbb{C})$ has a unique irreducible representation $\rho_{n}$ of dimension $n=2,3, \ldots$ For the Casimir we have

$$
\rho_{n}(\Delta)=n^{2}-1
$$

So we see that for finite-dimensional representations, the Casimir can take only a discrete set of values. This explains why only for some discrete set of $\lambda$ 's, there exist finite-dimensional modules for $\mathrm{gl}(\lambda)$. This gives an algebraic explanation for the "quantization of cohomology" as discussed in [7], though it does not explain why only for $\lambda \in\{1,2,3,4, \ldots\}$ there is a module of smooth functions, namely $\mathbb{P}_{n}$.

\section{The Structure of $\mathrm{gl}(\lambda)$}

In this section we return to considering $\operatorname{gl}(\lambda)$ as space of differential operators.

4.1.

By construction, $\operatorname{gl}(\lambda)$ contains $\operatorname{sl}_{2}(\mathbb{C})$ as a subalgebra, $\operatorname{sl}_{2}(\mathbb{C})$ being $\langle e, h, f\rangle$. Hence we can consider $\operatorname{gl}(\lambda)$ as an $\operatorname{sl}_{2}(\mathbb{C})$-module. Let $\operatorname{gl}(\lambda)^{(n)}$ denote all elements of $\operatorname{gl}(\lambda)$ up to order $n$. In particular, we have that $\operatorname{gl}(\lambda)^{(1)}$ is a Lie subalgebra isomorphic to $\mathrm{gl}_{2}(\mathbb{C})$, and $\mathrm{gl}(\lambda)^{(1)} / \mathrm{gl}(\lambda)^{(0)}$ is just $\mathrm{sl}_{2}(\mathbb{C})$. More general, for all $n \in \mathbb{N}, \operatorname{gl}(\lambda)^{(n)}$ is a finite-dimensional $\operatorname{sl}_{2}(\mathbb{C})$-module. At first sight the order could be $n+1$, but the commutator reduces one order. By Weyl's theorem, $\operatorname{gl}(\lambda)^{(n)}$ is completely reducible as an $\mathrm{sl}_{2}(\mathbb{C})$-module. We choose a basis, reflect- 
ing this fact. This basis will contain the elements $\left\{p^{i} q^{j}\right\}, i, j \in \mathbb{N}, i+j \in 2 \mathbb{N}$. If $i=0(j=0)$, we just write $q^{j}\left(p^{i}\right)$. We define $\left\{p^{i} q^{j}\right\}$ inductively by

$$
p^{2 n}=2 e^{n} \quad \text { and } \quad p^{i} q^{j}=-\frac{1}{i+1}\left[\partial, p^{i+1} q^{j-1}\right] .
$$

A straightforward calculation yields the following form for $p^{i} q^{j}, i+j=2 n$.

LEMMA 4.1.

$$
p^{i} q^{j}=(-1)^{i}\left\{2 x^{i} \partial^{n}+i(n-\lambda) x^{i-1} \partial^{n-1}+\left(\begin{array}{l}
i \\
2
\end{array}\right) \gamma(n) x^{i-2} \partial^{n-2}+\cdots\right\},
$$

where

$$
\gamma(n)=\frac{n-1}{2 n-1}\left\{(n-1)(n-2)+(2 n-3)(1-\lambda)+(1-\lambda)^{2}\right\} .
$$

4.2 .

Now we consider the commutator structure of $\mathrm{gl}(\lambda)$. Till now it is not clear why we denoted the special basis by $\left\{p^{i} q^{j}\right\}$, but the following proposition will clarify this.

PROPOSITION 4.2. Let $\left\{p^{i} q^{j}\right\}$ denote the basis of $\mathrm{gl}(\lambda)$ as introduced above. Then

$$
\left[p^{i} q^{j}, p^{k} q^{l}\right]=(i l-j k) p^{i+k-1} q^{j+l-1}+\sum_{s=2}^{\infty} c_{s}\left(p^{i} q^{j}, p^{k} q^{l}\right)
$$

where $c_{s}: \operatorname{gl}(\lambda) \times \operatorname{gl}(\lambda) \rightarrow \operatorname{gl}(\lambda)$ maps $\operatorname{gl}(\lambda)^{(m)} \times \operatorname{gl}(\lambda)^{(n)}$ to $\operatorname{gl}(\lambda)^{(m+n-s-1)}$, and $c_{s}$ is degree-preserving, meaning that

$$
\operatorname{deg}\left(c_{s}\left(p^{i} q^{j}, p^{k} q^{l}\right)\right)=\operatorname{deg}\left(p^{i} q^{j}\right)+\operatorname{deg}\left(p^{k} q^{l}\right) .
$$

Moreover

$$
c_{2}=\frac{1}{4}\left(\lambda^{2}-\frac{1}{4}\right) c_{2}^{\prime \prime}-\frac{1}{48} c_{2}^{\prime}
$$

with

$$
\begin{aligned}
& c_{2}^{\prime}\left(p^{i} q^{j}, p^{k} q^{l}\right) \\
&= k(k-1)(k-2) j(j-1)(j-2)-i(i-1)(i-2) l(l-1)(l-2)+ \\
& \quad+3 i j k l(l i-j k+k+j-i-l) p^{i+k-3} q^{j+l-3}
\end{aligned}
$$

and

$$
c_{2}^{\prime \prime}\left(p^{i} q^{j}, p^{k} q^{l}\right)=\frac{c_{2}^{\prime}\left(p^{i} q^{j} \wedge p^{k} q^{l}\right)}{(k+l-1)(i+j-1)(i+j+k+l-3)}
$$

and for all $s=2,3, \ldots$

$$
c_{s}\left(p^{i} q^{j}, p^{k} q^{l}\right)=0 \text { for } i+j \leqslant 2 .
$$


The proof of this proposition is straightforward (though tedious), using the explicit form of $p^{i} q^{j}$ from Lemma 4.1. The last statement is a reformulation is the $\mathrm{sl}_{2}(\mathbb{C})$-structure, which motivated the choice of the $\left\{p^{i} q^{j}\right\}$. From this proposition one sees that $\operatorname{gl}(\lambda)$ is a deformation of a Poisson subalgebra on the plane. This aspect will be discussed in Section 5.

4.3 .

We will draw some conclusions from the foregoing. The next lemma shows how natural the choice of the $\left\{p^{i} q^{j}\right\}$ is.

LEMMA 4.3. Let $I \subset \operatorname{sl}(\lambda)$ be an ideal. Then either $I=0$ or $I$ contains all $p^{i} q^{j}$ with $i+j \geqslant 2 n$ for some $n \in \mathbb{N}$.

Proof. If $I$ is nonzero, it contains an element, say $x \neq 0$. We may assume that $x$ is graded, since acting by $h$ decomposes $x$. So suppose $x$ has degree $m$ and order $n$ :

$$
x=\sum_{i=0}^{n} a_{i} p^{i+m} q^{i-m}, \quad a_{n} \neq 0 .
$$

By $n-m$ times applying $p^{2}$ to $x$, we see that $p^{2 n} \in I$. By $i$ times applying $q^{2}$ to $p^{2 n}(i=1,2, \ldots, 2 n)$, we see that $p^{2 n-i} q^{i} \in I$. Similarly, by applying $p^{3} q$ to $p^{2 n}$, it follows that also $p^{2 n+2} \in I$. Continuing this process, we see that all $p^{i} q^{j}$ with $i+j \geqslant 2 n$ are in $I$. If $I$ contains elements outside this linear space, we can continue till this is no longer the case.

Now we are able to prove simplicity for almost all $\operatorname{sl}(\lambda)$.

PROPOSITION 4.4. sl $(\lambda)$ is simple if and only if $\lambda \notin\{ \pm 2, \pm 3, \pm 4, \ldots\}$.

Proof. The only if-part we proved in Subsection 2.3. So we only need to prove that $\operatorname{sl}(\lambda)$ is simple for $\lambda \notin\{ \pm 2, \pm 3, \pm 4, \ldots\}$. So take such a $\lambda$, and suppose $I$ is a nontrivial ideal. Let $x$ be a minimal (in order) element in $I$, say $\operatorname{ord}(x)=n$. If $n=1$ then $I=\operatorname{sl}(\lambda)$ (again by Lemma 4.3), so we may assume that $n \geqslant 2$. According to Lemma $4.3, I$ contains $p^{2} q^{2 n-2}$. Now one can calculate the two leading terms in $\left[p^{4}, p q^{2 n-1}\right]$ using Proposition 4.2:

$$
\left[p^{4}, p q^{2 n-1}\right]=(8 n-4) p^{4} q^{2 n-2}+\alpha\left(\lambda^{2}-n^{2}\right) p^{2} q^{2 n-4}+\cdots,
$$

where $\alpha \neq 0$. So we see, since $\lambda^{2}-n^{2} \neq 0$ for $\lambda \notin\{ \pm 2, \pm 3, \pm 4, \ldots\}$, that $p^{2} q^{2 n-4}+\cdots \in I$. But this contradicts the minimality of $x$.

\section{5. $\operatorname{gl}(\lambda)$ as Deformation of a Poisson Algebra}

In the previous section, we noted that $\operatorname{gl}(\lambda)$ is a deformation of some Poisson algebra. Here we will discuss this correspondence in more detail. 


\section{1}

Let $H_{0}$ denote the subalgebra of the Poisson algebra on the plane, consisting of even polynomials: a basis for $H_{0}$ is $\left\{p^{i} q^{j}\right\}$, with $i, j \in \mathbb{N}, i+j \in 2 \mathbb{N}$, and the commutator is given by

$$
\left[p^{i} q^{j}, p^{k} q^{l}\right]=(i l-j k) p^{i+k-1} q^{j+l-1} .
$$

As one sees from Proposition 4.2, the commutators of $H_{0}$ and $\operatorname{gl}(\lambda)$ agree on top level. However in $H_{0}$ there are no terms of lower order. Therefore the order is not only a filtering on $H_{0}$, but even a grading*.

In Feigin's paper [4], one of the central problems is to find all deformations of $H_{0}$. Here we take deformations to mean deformations which leave $H_{0}$ on top-level unaltered. So a deformation will mean a Lie algebra with basis $\left\{p^{i} q^{j}\right\}$, $i+j$ even and Lie product $[\cdot, \cdot]_{t}$ of the form

$$
\begin{aligned}
{\left[p^{i} q^{j}, p^{k} q^{l}\right]_{t}=} & (i l-j k) p^{i+k-1} q^{j+l-1}+ \\
& +\sum_{r+s=2,4, \ldots} t^{r+s} a(i, j, k, l, r, s) p^{i+k-1-r} q^{j+l-1-s} .
\end{aligned}
$$

Some deformations can, by a change of basis, be transformed to the original Lie algebra, i.e. after a change of basis we find $\tilde{p}^{i} \tilde{q}^{j}$ such that $\tilde{a}(i, j, k, l, r, s)=0$. Such deformations are called trivial. Using the technique of inner gradings (see $[4], \S 1.5 .2)$, one knows that any deformation of the form above can be put in the form

$$
\left[p^{i} q^{j}, p^{k} q^{l}\right]_{t}=(i l-j k) p^{i+k-1} q^{j+l-1}+\sum_{s=2}^{\infty} t^{2 s} c_{s}\left(p^{i} q^{j}, p^{k} q^{l}\right)
$$

with $c_{s}\left(p^{i} q^{j}, p^{k} q^{l}\right)=a(i, j, k, l, s) p^{i+l-1-s} q^{j+l-1-s}$. Moreover one can prove that any nontrivial deformation can be put in a form such that the first appearing nonvanishing $c_{s}$ is nonzero in $H_{(-s)}^{2}\left(H_{0} ; H_{0}\right)$, the second cohomology group of $H_{0}$ with coefficients in the adjoint representation. Therefore, to find nontrivial deformations it is necessary to find $H_{(-s)}^{2}\left(H_{0} ; H_{0}\right)$. This calculation can be performed by computer using spectral sequences, and thanks to the fact that $H^{2}\left(H_{0}\right)$ is known (see [8]). Here we only give the result. For $s \geqslant 1$, we find $H_{(-s)}^{2}\left(H_{0} ; H_{0}\right)=0$ unless $s=2$, and a basis of $H_{(-s)}^{2}\left(H_{0} ; H_{0}\right)$ is $\left\{c_{2}^{\prime}, c_{2}^{\prime \prime}\right\}$ as defined in Proposition 4.2. We note that $c_{2}^{\prime}$ can be extended to the full Poisson algebra, and is well-known there, see, for example, [6, Section 11]. Extension to the full Poisson algebra is impossible for $c_{2}^{\prime \prime}$.

\section{2.}

Our problem was to find all deformations of $H_{0}$ in the sense of the previous subsection. We know that for the first term we can take a linear combination

\footnotetext{
* The degree being one less than the order, i.e the degree of $p^{i} q^{j}$ is $(i+j) / 2-1$.
} 
of $c_{2}^{\prime}$ and $c_{2}^{\prime \prime}$. This first term is called the infinitesimal deformation. Usually not all infinitesimal deformations can be extended to a deformation. In this case, however, the situation is different. This is not difficult to prove: going from $\alpha c_{2}^{\prime}+\beta c_{2}^{\prime \prime}$ to $\gamma\left(\alpha c_{2}^{\prime}+\beta c_{2}^{\prime \prime}\right)$ corresponds to rescaling $t$. Hence, effectively the (infinitesimal) deformations are parameterized by $\mathbb{P}_{1}(\mathbb{C})$ instead of $\mathbb{C}^{2}$. But for all elements $(\alpha: \beta) \in \mathbb{P}_{1}(\mathbb{C})$ except $(0: 1)$ we have already a deformation, namely $\operatorname{gl}(\lambda)$, where $\lambda$ is such that $\left(-\frac{1}{48}:-\frac{1}{4}\left(\lambda^{2}-1\right)\right)=(\alpha: \beta)$. So all infinitesimal deformations $\alpha c_{2}^{\prime}+\beta c_{2}^{\prime \prime}$ can be extended to a deformation, except possibly $c_{2}^{\prime \prime}$. But the extendible values $(\alpha, \beta)$ form a closed set in $\mathbb{C}^{2}$. So also $c_{2}^{\prime \prime}$ can be extended. We discuss this case in the next subsection.

We close this subsection with a final remark. One can prove that all $c_{s}, s=$ $1,2,3, \ldots$ in $(5.1)$ can be chosen $\mathrm{sl}_{2}(\mathbb{C})$-invariant, i.e.

$$
\left[g, c_{s}(x, y)\right]=c_{s}([g, x], y)+c_{s}(x,[g, y])
$$

for $g \in \mathrm{sl}_{2}(\mathbb{C})=\langle e, h, f\rangle$. However one can also prove that there are no $\mathrm{sl}_{2}(\mathbb{C})$ invariant coboundaries in $B_{(-s)}^{2}\left(H_{0} ; H_{0}\right)$. Since the nonuniqueness in the $c_{s}$ is exactly the adding of an element of $B_{(-s)}^{2}\left(H_{0} ; H_{0}\right)$, it follows that the series $\left(c_{s}\right)$ takes a unique form, if we require $\mathrm{sl}_{2}(\mathbb{C})$-invariance. For a more detailed discussion, see [9].

\section{3.}

Here we discuss the deformation corresponding to $c_{2}^{\prime \prime}$. We give an explicit description of this algebra. It turns out that this algebra is the only one among the deformations, which can be realized linearly. By this we mean, that by taking a suitable representative for $\frac{1}{8} c_{2}^{\prime \prime}$, we find that for the series $\left(c_{n}\right)$ satisfies $c_{1}=c_{3}=c_{4}=\cdots=0$. The deformation corresponding to $\frac{1}{8} c_{2}^{\prime \prime}$ is

$$
\left[p^{i} q^{j}, p^{k} q^{l}\right]_{t}=(i l-j k) p^{i+k-1} q^{j+l-1}+t^{2} c_{2}\left(p^{i} q^{j}, p^{k} q^{l}\right)
$$

where

$$
\begin{aligned}
& c_{2}\left(p^{2 k+1} q^{2 r+1}, p^{2 l+1} q^{2 s+1}\right)=(k s-r l) p^{2 k+2 l-1} q^{2 r+2 s-1}, \\
& c_{2}\left(p^{2 k} q^{2 r}, p^{2 l+1} q^{2 s+1}\right)=(k s-r l) p^{2 k+2 l-2} q^{2 r+2 s-2}, \\
& c_{2}\left(p^{2 k} q^{2 r}, p^{2 l} q^{2 s}\right)=0 .
\end{aligned}
$$

By a direct calculation one can show that $c_{2}=\frac{1}{8} c_{2}^{\prime \prime}$ modulo some coboundary. This coboundary is chosen such that the Massey square (see [5]) of $c_{2}$ is 0 . However, $c_{2}$ is not $\operatorname{sl}_{2}(\mathbb{C})$-invariant any more.

\section{4 .}

Finally some words about defining relations for $\operatorname{gl}(\lambda)$. Thanks to [8], we know a set of defining relations for $H_{0}$ : if the commutators between the elements $\left\{p^{i} q^{j}\right\}$ 
for $i+j \leqslant 4$ and $\left\{p^{k} q^{l}\right\}$ for $k+l \leqslant 6$ are given ${ }^{\star}$, then the corresponding Lie algebra (i.e. the quotient of the free Lie algebra on the generators $\left\{p^{i} q^{j}\right\}$ for $i+j \leqslant 6$ modulo the ideal of relations) is isomorphic to $H_{0}$.

Now all $\operatorname{gl}(\lambda)$ have $H_{0}$ as the associated graded Lie algebra. From this fact it is not difficult to prove, that in this case the commutators of $\left\{p^{i} q^{j}\right\}$ for $i+j \leqslant 4$ and $\left\{p^{k} q^{l}\right\}$ for $k+l \leqslant 6$ are also defining relations.

\section{Relation Between $\mathrm{gl}(n+1)$ and $\operatorname{Inv}\left(\mathbb{P}_{n}\right)$}

6.1 .

In this section, we discuss the relation between $\operatorname{gl}(n+1)$ and $\operatorname{Inv}\left(\mathbb{P}_{n}\right)$. A part of this relation was already discussed in Proposition 2.2. Here we recall a theorem from [11].

THEOREM 6.1. Let $T$ be a differential operator of order less than $n+1$, and suppose that $T \in \operatorname{Inv}\left(\mathbb{P}_{n}\right)$. Then $T \in \operatorname{gl}(\lambda)$ with $\lambda=n+1$.

Note that terms $c x^{l} \partial^{k}$ for $k \geqslant n+1$ annihilate $\mathbb{P}_{n}$, so such terms are in $\operatorname{Inv}\left(\mathbb{P}_{n}\right)$. So the algebra $\operatorname{Inv}\left(\mathbb{P}_{n}\right)$ consists more or less of two (intersecting) parts: $\mathrm{gl}(\lambda)$ and differential operators $T$ of the form $T=\tilde{T} \partial^{n+1}$. Our purpose is to show how these parts can be united.

\section{2 .}

Suppose that $T$ has degree $m$. If $\operatorname{deg}(T) \leqslant 0$ then automatically $T \in \operatorname{Inv}\left(\mathbb{P}_{n}\right)$, but also $T \in \operatorname{gl}(n+1)$, see Subsection 2.3. So let us assume that $m>0$. It is clear that in this case $T$ should annihilate $x^{n}, x^{n-1}, x^{n-2}, \ldots, x^{n-m+1}$. Here we see the reason for the "break" at $n+1$ : if $m>n+1$, then $n-m+1<0$, and in the series above the last terms disappear. So it seems that the following number should be important:

$$
p(m)=|\{i \in\{0,1,2, \ldots, n\} \mid i+m>n\}| .
$$

To prove the main proposition, we need the following remarkable result from [12].

LEMMA 6.2. For all $n=0,1,2, \ldots$ there holds

$$
\left(x^{2} \partial-n x\right)^{n+1}=x^{2 n+2} \partial^{n+1} .
$$

* Which means that we consider $\left\{p^{i} q^{j}\right\}$ for $i+j=8$ as formal elements; the first time such an element appears, can be considered as the definition of it, all next times are relations. 
Proof. It is clear that $\left(x^{2} \partial-n x\right)^{n+1}=x^{2 n+2} \partial^{n+1}+\cdots$. However, $\left(x^{2} \partial-\right.$ $n x)^{n+1}$ annihilates $\mathbb{P}_{n}$. Therefore the dots vanish, since otherwise $\mathbb{P}_{n}$ is not annihilated.

The number $p(m)$ occurs in the following proposition:

PROPOSITION 6.3. Suppose $T$ has degree $m, e=x^{2} \partial-n x(\in \operatorname{gl}(n+1))$ and $T \in \operatorname{Inv}\left(\mathbb{P}_{n}\right)$. Then there exists a differential operator $\widetilde{T}$ such that

$$
T=\widetilde{T} \mathrm{e}^{p(m)} \text {. }
$$

Proof. If $m \leqslant 0$, there is nothing to prove. We assume that $T$ has order $k$, so that we have $T=\alpha x^{m+k} \partial^{k}+\cdots, \alpha \neq 0$.

(1) First we consider the case that $m>k$. Since $\operatorname{Inv}\left(\mathbb{P}_{n}\right)$ is an algebra, $[e, T]=$ $(m-k) x^{m+k+1} \partial^{k}+\cdots \in \operatorname{Inv}\left(\mathbb{P}_{n}\right)$ and continuing we find that $\operatorname{Inv}\left(\mathbb{P}_{n}\right)$ would contain an element $\bar{T}=x^{n+1+k} \partial^{k}+\cdots$. If, however $k \leqslant n$ this is impossible, since then $\bar{T}$ does not annihilate $\mathbb{P}_{n}$. So there are no elements $T$ in $\operatorname{Inv}\left(\mathbb{P}_{n}\right)$ with $\operatorname{deg}(T)>\operatorname{ord}(T)$, if $\operatorname{ord}(T) \leqslant n$. On the other hand, if $T=x^{m+k} \partial^{k}$ with $k \geqslant n+1, m \geqslant k$, one can find $\tilde{T} \in \mathfrak{D}$ such that $T=\widetilde{T} x^{2 n+2} \partial^{n+1}$. But $\left(x^{2} \partial-n x\right)^{n+1}=x^{2 n+2} \partial^{n+1}\left(\right.$ Lemma 6.2), so indeed $T=\widetilde{T} e^{n+1}=\widetilde{T} e^{p(m)}$.

(2) To prove (6.1) for all $m, k$ we apply induction with respect to $k$, keeping $m$ fixed. From the previous (1) we know that $(6.1)$ is tnue for $m>k$, so for small $k$ our hypothesis is true. So assume now that $T=\alpha x^{m+k} \partial^{k}+\cdots$. If $k<m$ there is nothing to prove, according to (1). So assume $k \geqslant m$. Now $\alpha x^{k-m} \partial^{k-m} e^{m}=\alpha x^{m+k} \partial^{k}+\cdots$ Clearly $\alpha x^{k-p(m)} \partial^{k-p(m)} e^{p(m)} \in \operatorname{Inv}\left(\mathbb{P}_{n}\right)$, so also $T-\alpha x^{k-p(m)} \partial^{k-p(m)} e^{p(m)} \in \operatorname{Inv}\left(\mathbb{P}_{n}\right)$. But, due to our induction hypothesis, we have that the last expression is a left multiple of $e^{p(m)}$. So also $T$ itself. This completes the proof.

It is not difficult to match Theorem 6.1 and Proposition 6.3. One other point is worth mentioning. Similar results as in Proposition 6.3 for $\mathbb{P}_{n}$ can be generalized to $\operatorname{Inv}(V)$ for $V=\left\langle x^{i_{1}}, x^{i_{2}}, \ldots, x^{i_{n}}\right\rangle$, see [10].

\section{Acknowledgement}

The authors wish to thank D. Leites and V. Serganova for stimulating discussions on $\operatorname{gl}(\lambda)$.

\section{References}

1. Bordemann, M., Hoppe, J., and Schaller, P.: Infinite dimensional matrix algebras, Phys. Lett. B 232(2) (1989), 199-203.

2. Dixmier, J.: Quotients simples de l'algèbre enveloppante de sl, J. Algebra 24 (1973), 551-564.

3. Dixmier, J.: Enveloping Algebras, North-Holland Math. Library 14, North-Holland, Amsterdam, 1977.

4. Feigin, B. L.: The Lie algebra $g l(\lambda)$ and cohomologies of Lie algebras of differential operators, Russian Math. Surveys 43(2) (1988), 157-158. 
5. Fuchs, D. B.: Cohomology of Infinite-Dimensional Lie Algebras, Plenum, New York, 1986.

6. Gerstenhaber, M. and Schack, S.: Algebraic cohomology and deformation theory, in M. Hazewinkel and M. Gerstenhaber (eds), Deformation Theory of Algebras and Structures and Applications, NATO ASI Series C, 247, Kluwer, Dordrecht, 1988.

7. González-López, A., Hurtubise, J., Kamran, N., and Olver, P.: Quantification de la cohomologie des algèbres de Lie de champs de vecteurs et fibrés en droites sur des surfaces complexes compactes, Preprint, 1992.

8. Van den Hijligenberg, N. and Post, G.: Defining relations for Lie algebras of vector fields, Indag. Math. N.S. 2(2) (1991), 207-218.

9. Post, G. and Van den Hijligenberg, N.: Explicit results on $\operatorname{gl}(\lambda)$ and $\operatorname{sl}(\lambda)$, Memorandum 1075, University of Twente, 1992.

10. Post, G. and Turbiner, A.: Classification of linear differential operators with an invariant space in monomials, Memorandum 1143, Faculty of Applied Mathematics, University of Twente, 1993.

11. Turbiner, A.: Lie-algebras and polynomials in one variable, J. Phys. A 25 (1992), L1087L1093.

12. Turbiner, A. and Post, G.: Operator identities, representations of algebras and the problem of normal ordering, J. Phys. A 27 (1994), L9-L13. 